\title{
Rechtsgeschichte
}

http://www.rg-rechtsgeschichte.de/rg1

Zitiervorschlag: Rechtsgeschichte Rg 1 (2002)

$\operatorname{Rg} 12002 \quad 306-308$

http://dx.doi.org/10.12946/rg01/306-308

\section{Heinrich Gehrke}

Schnappschüsse im Gerichtssaal 


\section{Schnappschüsse im Gerichtssaal}

Öffentlichkeit der Verhandlung ist eine der obersten Maximen unserer Strafprozesse. So mühsam diese Forderung im Geist der Aufklärung vom bürgerlichen Liberalismus vor 200 Jahren erkämpft wurde, so hoch wird der heilige Grundsatz - »das Kernstück des Strafverfahrens « (so Pfeiffer in der Einleitung des Karlsruher Kommentars zur StPO) - noch heute, meilenweit von der Geheimjustiz des Absolutismus entfernt, gehalten. In peniblen Entscheidungen hat sich die höchstrichterliche Rechtsprechung über Generationen mit so zentralen Fragen wie der auseinandergesetzt, ob ein versehentliches Abschließen einer Gerichtstür oder ein unzulänglicher Hinweis auf den Verhandlungssaal zur Urteilsaufhebung nach $\$ 338 \mathrm{Nr}$. 6 StPO (Verletzung der Öffentlichkeit) führen muss - und dabei die doch wohl recht beschränkte praktische Relevanz des Publizitätsprinzips etwas aus den Augen verloren oder überbewertet.

Bei etwas genauerer Betrachtung gilt dieses Prinzip - wie es solche Grundsätze nun mal so an sich haben - nämlich nur "prinzipiell«: Es bezieht sich in direkter Anwendung lediglich auf die (wenigen) Bürger, die sich rechtzeitig am Gerichtsort ein- und dort auch Platz gefunden haben. Die müssen - ein zweifelhaftes Mandat ihre Mitbürger (und damit die Öffentlichkeit) »vertreten «. Ob und wie sie es tun, darum kümmert sich niemand. Ebenso fragt sich niemand im Zeitalter des strafprozessualen "Deals", welche rechtsstaatlich notwendige Kontrollfunktion diese wenigen Zuschauer noch der Justiz gegenüber ausüben können.

Gemach, sagt da so mancher, alles nicht so schlimm: Wir haben da ja noch die Medien, die mit ihrer Justizberichterstattung das Wächter- amt wahrnehmen und die breite Öffentlichkeit darüber informieren, wie es in deutschen Gerichtssälen zugeht. Lassen wir dies einmal so stehen, verdrängen wir beim Gedanken an Gerichtsreportagen unweigerlich aufkommende Reizthemen wie Korrektheit, Sachlichkeit, Sensationslust, so bleibt doch eines durchaus eigenartig: Obwohl Presse, Funk und Fernsehen notwendigerweise die wesentliche Mittlerfunktion zwischen Gerichtsgeschehen und Öffentlichkeit übernommen haben, obwohl ohne sie die Justiz sich doch praktisch »hinter verschlossenen Türen abspielen " (BGHSt 7, 22I) würde, sind ihnen seit 1965 die in unserer Mediengesellschaft wichtigsten Informationsmethoden - Ton-, Filmund Fernsehaufnahmen aus der Verhandlung untersagt. Mag auch das Bundesverfassungsgericht in letzter Zeit in einigen richtungsweisenden Entscheidungen (Honecker, Al-Qaida) die Grenzen für den Ausschluss bewegter Bildaufnahmen etwas enger gezogen haben, am Grundsatz, dass nur die im Gerichtssaal Anwesenden das echte Geschehen, die wahre Atmosphäre, das wirkliche Agieren aller Beteiligten erleben können, hat sich nichts geändert. Unschuldsversichernd lächelnde oder ihr Gesicht verbergende Angeklagte, selbstbewusst und beflissen rechtskundig auftretende Verteidiger und Staatsanwälte, ein steif den Saal betretendes und bis zum Abschalten der Scheinwerfer herumstehendes Gericht - das ist alles, was die Öffentlichkeit auch von wichtigsten Strafprozessen im Fernsehen zu sehen bekommt.

Und genau das war die Basis für das selbstgesetzte Berufsziel des I916 geborenen, I999 (I20 Jahre nach Honoré Daumier) im Alter von 83 Jahren gestorbenen Zeichners und

- Erich Dittmann, »Nieder mit den schwarzen Paragrafenhengsten!« Zeichnungen aus deutschen Gerichten 1963-I999, hg. von Thomas Lang, München: Beck 200I, ISBN 3-406-48587-I 
Portraitisten Erich Dittmann. Als erster, jedenfalls als bekanntester der viel zu wenigen Künstler seines Genres, die sich im Nachkriegsdeutschland dieser Aufgabe gewidmet haben, füllte er diese rechtliche Lücke von ihrem Entstehen an mit seinem genialen Zeichenstift. Schon früh konnte er in der Frankfurter Allgemeinen Zeitung seine lebendigen Momentaufnahmen des Prozessgeschehens veröffentlichen, und er tat dies dort und in anderen wichtigen Zeitungen bis unmittelbar vor seinem Tod. In so gut wie allen wichtigen bundesdeutschen Strafverfahren des letzten Jahrhundertdrittels war er zugegen, und wer der daran Beteiligten kannte ihn nicht, den stillen, unauffälligen kleinen Herrn, der meist einen kleinen Platz vor den übrigen Pressevertretern, näher an den Akteuren, beanspruchen durfte und dessen Anwesenheit bewirkte, dass so mancher sich - seines Portraitiertwerdens bewusst - vorteilhaft »ins Bild zu setzen« versuchte.

Eine charakteristische Auswahl seiner Gerichtszeichnungen aus den 36 Jahren von Ende I 963 bis 1999 ist jetzt bei C. H. Beck erschienen, angemessener Weise bei einem juristischen Verlag, denn es ist - zeitgenössische - Rechtsgeschichte, die sich dem Leser beim Betrachten der Bildersammlung darbietet. Die historische Bedeutung der Justizdokumentation zeigt sich auch daran, dass sie aus den rund 500 Grafiken und ähnlichen Materialien zusammengestellt wurde, die Dittmann I995 dem Bonner »Haus der Geschichte der Bundesrepublik Deutschland « zur Verfügung gestellt hat, dessen Sammlungsleiter Dietmar Preißler sie mit einer sehr einfühlsamen Einführung versehen hat.

Blättert man als jemand, der das deutsche Gerichtsgeschehen des ausgehenden 20. Jahrhunderts miterlebt und -erlitten hat, durch die chronologisch geordnete Sammlung, so revitali- siert sich spontan die Erinnerung an damals schmerzvoll, empört, betroffen, engagiert, amüsiert oder einfach nur interessiert verfolgte große Prozesse, die ein Spiegelbild des jeweiligen Zeitgeschehens waren. Das beginnt 1963 mit dem großen Frankfurter Auschwitz-Verfahren, springt dann - ein wenig zu abrupt - zum zwölf Jahre später begonnenen Stammheimer Baader-Meinhof-Prozess, führt über viele andere Verhandlungen gegen Staatsgegner (Linksradikale, Hausbesetzer, Atomkraft- und Startbahnopponenten, RAF und andere Terroristen) und Spione (Guillaume, Ehepaar Lutze, Cremer u. a.), schwenkt nach der Auflösung von RAF und DDR zur juristischen Aufarbeitung von deren "Nachlass « (Aburteilung der »reuigen « Aussteiger, im Osten untergetauchten Ex-Terroristen und ihrer StasiBeschützer), spiegelt die neue Bedrohung durch die Globalisierung von Konflikten und Terror (Hamadi, Mykonos, PKK) und endet - ein Zeichen wiedererlangter Normalität? - bei großen Wirtschafts- und Mordprozessen (Lucona, Dr. Schneider, Monika Weimar).

Alles in allem scheinen mir die Zeichnungen aus Verfahren mit politischem Einschlag doch recht überrepräsentiert. Von den rund hundert Grafiken der Sammlung stammen aus Verfahren gegen die linke Szene (bis zu den Terroristen von RAF und Konsorten) ein gutes Drittel, mit den Bereichen Spionage, Alt- und Neonazis, ausländische Konflikte und »Nach-Wende «-Prozesse (Schalck-Golodkowski u. a.) rund zwei Drittel. Dagegen kann allenfalls ein Viertel dem »ordinären « Kriminalitätsgeschehen (Kapitaldelikte, Entführung, Wirtschaftsverbrechen, Kinderschänder, Parteispenden) zugeordnet werden. Ganz so "politiklastig « war die spektakuläre Strafprozessgeschichte in jenem Zeitraum wohl doch nicht, die Auswahl mag an der HistorikerOptik des Bonner Mitherausgebers liegen.

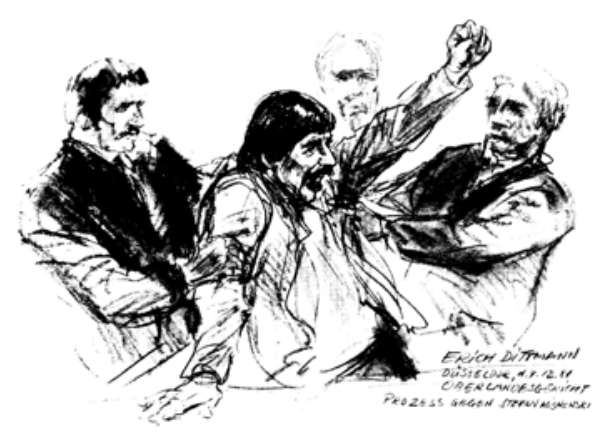


Aber das ist kein Manko. In diesem Kontext geboten wird ein lebendiges, informatives Kaleidoskop aus dem Zentrum der einzelnen Verfahren. Die Grafiken und Skizzen fassen meisterhaft das für den stillen Beobachter mit dem Zeichenstift wesentliche und typische von Prozessgeschehen und beteiligten Personen zusammen, teils nur schemenhaft hingeworfen, teils bis ins Detail ausgearbeitet. »Ganz objektiv und neutral « sah sich Dittmann in seinen Abbildungen, aber dies war wohl nur eine fromme Selbstverkennung. Zwar hat er, anders als sein großer Vorgänger Daumier, keine bewusst provozierenden, vom Einzelfall gelösten Justizkarikaturen geschaffen, aber sein subjektives Engagement, sein Miterleben und -erleiden des Verhaltens von Tätern und Opfern vor Gericht hat ihn doch oft $\mathrm{zu}$ emotional »überzeichneten « - und gerade dadurch noch heute so mitreißend-lebendigen Bildprotokollen von Menschen und Szenen geführt. Auch die notwendige Entscheidung des Zeichners für eine den jeweiligen Prozess typisch spiegelnde Verhandlungssituation ist ja nicht ohne Subjektivität zu treffen, und wer weiß, wie langweilig es auch in Sensationsprozessen über lange Zeit zugehen kann, muss den Künstler für seine Auswahl bewundern.

Wer von vielen der Verfahren altersbedingt wenig gehört bzw. - verständlich in unserer schnelllebigen Zeit - doch so manches vergessen hat, kann sich durch die zu den ausgewählten 70 Strafverfahren gegebenen kurzen, treffenden und (bei dem politischen Übergewicht der Auswahl keine Selbstverständlichkeit!) wirklich objektiven Informationen über die wesentlichen Prozessdaten rasch kundig machen bzw. seiner Erinnerung aufhelfen. $\mathrm{Zu}$ den einzelnen Zeichnungen finden sich zusätzlich spezielle Erläuterungen der jeweils festgehaltenen Szene, oft basierend auf Kommentierungen und markanten
Aussprüchen Prozessbeteiligter, die Dittmann auf seinen Blättern festgehalten hat (unter anderem den programmatischen Ausruf des Angeklagten Teufel vor dem Landgericht Berlin-Moabit, welcher dem Band seinen Titel gegeben hat).

So ist das kleine Buch eine kurzweilige, aber dennoch sehr informative Retrospektive auf eine noch gar nicht so lange zurückliegende bewegte Zeit deutscher Justizgeschichte. Schade, dass »Gerichtszeichner « wohl ein aussterbender Beruf ist, kann doch nur er angesichts unserer medienfeindlichen Rechtslage auch optisch das Prozessgeschehen lebendig vermitteln. *

Heinrich Gehrke

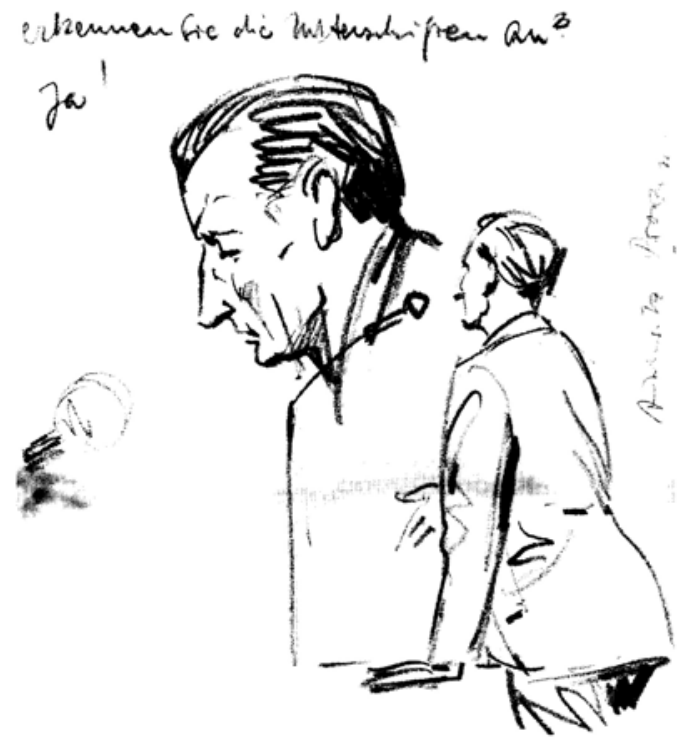

Die Abbildungen stammen aus dem besprochenen Band:

S. 307: Der Terrorist Stefan Wisniewski randaliert I98 I bei der Urteilsverkündung im SchleyerProzess, wo er sich als erster der Beteiligten vor Gericht rechtfertigen musste.

Diese Seite: Arthur Breitwieser im Frankfurter Auschwitz-Prozess.

ิ Hier erklärt er, er habe mit Zyk-

on B nur die Kleider desinfizieren wollen. Breitwieser wurde im Prozess freigesprochen. 